\title{
Design of Distributed Control System for Radiotherapy Equipment Based on Field Bus
}

\author{
S.H. Shi, X. Zhang \\ Department of Mechanical Engineering \\ Tsinghua University \\ China
}

\author{
P.Q. Ye \\ Beijing Key Lab of Precision /Ultra-precision \\ Manufacturing Equipments and Control \\ China
}

\author{
H. Zhang \\ The State Key Lab of Tribology \\ Tsinghua University \\ China
}

\begin{abstract}
Radiotherapy is one of the most important therapeutic methods for tumor. As more and more therapeutic methods been put forward, rigorous requirements have been set on the realtime performance, stability and maintainability of the radiotherapy equipment control system. This article designs a Four-Tier Distributed Control System with main control layer, sub control layer, execution layer and hardware control layer in accordance with the characteristics and requirements of radiotherapy equipment, as well as the entire hardware and control software of the system. Sample machine test shows that all subsystems are of stable operation and good performance, and real-time performance and all accuracy indexes fully meet the requirements of radiotherapy.
\end{abstract}

Keywords: radiotherapy equipment; distributed; field bus; control system

\section{INTRODUCTION}

With the development of science and technology, more and more therapeutic methods to improve the radiation therapy effects have been put forward [1]. For example, using Electronic Portal Imaging Device (EPID) system to assist patients positioning, and using Image-Guided Radiation Therapy (IGRT) system or three-dimensional motion detection equipment to track patients' respiration [2,3]. Meanwhile, for some clients, several functions may need to be deleted [4]. Centralized control system [5] is normally used in the current radiotherapy equipments, which will negatively influence the real-time performance when there are too many signals to be processed. Therefore, a new structure of radiotherapy equipment control system needs to be designed, with which functional modules can be easily added or removed, damaged modules can be conveniently replaced, and cycle time is very short [6].

In order to find solutions for this problem, this article builds a Distributed Control System (DCS) based on the functions, characteristics and requirements of radiotherapy equipment. The whole control system is divided into four layers including main control layer, sub control layer, execution layer and hardware control layer. Every independent functional module is controlled separately so that signals from lower levels can be responded and processed quickly. Pressure on the main controller can be diverted and the real-time performance of the control system can be improved. The design also makes it easy for the system maintenance and functional module changing.

\section{OVERALL DESIGN OF THE DCS FOR RADIOTHERAPY EQUIPMENT}

Besides the base function components such as gantry, treatment couch, head and accelerators, in order to detect respiratory motion, the radiotherapy equipment in this article also has an IGRT system and an EPID system. The equipment uses bi-directional rotation radiotherapy. It contains 13 rotary motors and 128 linear Motors attaching to Multi Leaf Collimator (MLC). The therapy aperture is $1 \mathrm{~m}$. The maximum radiation field on central plane is $20 \times 40 \mathrm{~cm}$. The control system is required to realize the coordination among each functional module, response quickly to emergencies and ensure the safe and reliable operation of the equipment.

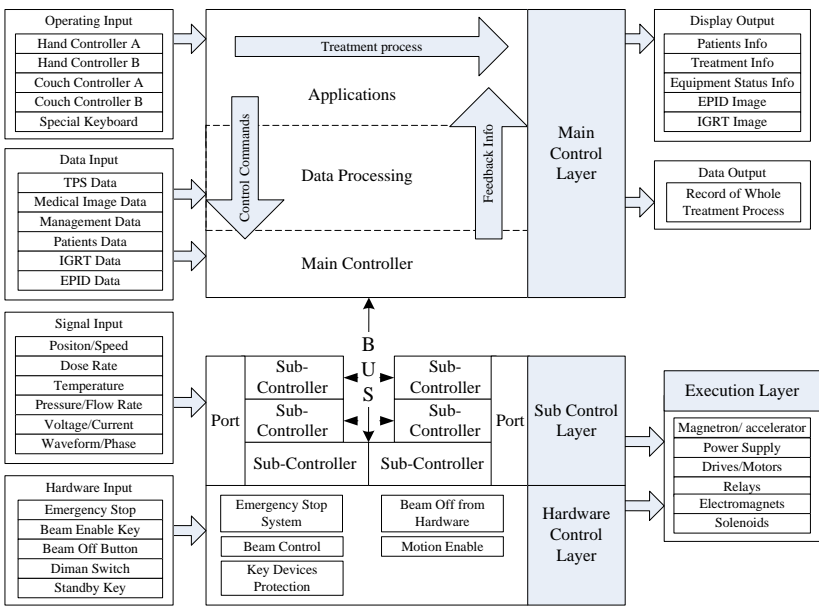

FIGURE I. STRUCTURE OF DCS FOR THE RADIOTHERAPY EQUIPMENT

In accordance to the structure of this radiotherapy equipment, the overall control system is designed as shown in Figure 1. The system includes four layers: main control layer, communication layer, sub-control layer and execution layer. The specific functions are as follows: 
(1) The main control layer consists of a workstation computer and a main controller (not refers to computer). It is the core of the whole control system. The workstation computer runs the applications and data processing program. The main controller receives commands from upper level programs and data information returned by sub-controllers, carries out kinematics calculation, trajectory planning, task scheduling and comprehensive decision on the radiotherapy process, and feeds back important data information to the upper level programs.

(2) The sub control layer includes 6 sub-controllers, each corresponding different functional modules. Sub-controllers directly process I/O and A/D signals from the bottom layer of each functional module and alarm signals from driver motor, and feed back important data and status to the main controller.

(3) The execution layer is responsible for completing the final driving motion of the equipment and collecting signals including rotary motors and linear motors generating motion of the equipment, digital outputs and analog outputs controlling magnetron, accelerator and relays, and digital inputs and analog inputs indicating speed, position, dose rate, temperature and flow rate.

(4) In addition, there is also a hardware control layer to take care of the signals requiring emergency response. These signals are not controlled by the software of the control system, but directly respond to appropriate hardware circuits.

\section{DETAILED DESIGN OF THE DCS FOR RADIOTHERAPY EQUIPMENT}

\section{A. Detailed Design of the Main Control Layer}

The applications of main controller layer are designed with four operation authorizations including morning check, therapy, physics and debugging for equipment maintenance personnel, therapist, radiation therapy physicist and research personnel respectively. With different operation authorization, the operators are provided with different working procedures and modification range on bottom layer parameters. Data processing program is on the bottom of the application program. The program should fully support the standard DICOM protocol so that information can be exchanged among any relevant medical equipment via network.

The main controller connects with the workstation computer on the upper layer via network cable to transmit commands and data, with each sub-controller on the bottom layer via field bus to send commands and receive current status, and with different Plug-in devices via interface protocol provided by equipment supplier such as connecting Image guidance system via cable and electronic portal imaging device via serial.

\section{B. Detailed Design of the Sub Control Layer}

1) Gantry sub-controller: Gantry sub-controller is responsible for gantry motion control and gantry status detection. It connects with two hand controller, which can be used to control the motion of all moving parts and the on and off of room lights, laser lights, field lights and distance lights. Via the field bus, the controller of the control room sends control commands to the gantry controller, and the gantry controller sends the information of the status of the gantry and the operation on hand controllers back to the control room.

2) Patient positioning sub-controller: The patient positioning sub-controller is in charge of the treatment couch motion control and treatment couch operation status detection. It also controls the movement and operation of the laser lights system to realize the positioning function by the coordination of the treatment couch and the laser lights system.

3) Thermostatic water sub-controller: When radiotherapy equipment is operating, large amount of heat will be generated from tokamak and magnetron. So the thermostatic water system's function is to take the heat away by using the circulation of distilled or deionized water.

Thermostatic water sub-controller is designed to detect work status of all the functional components (pumps, cooling fans, heaters, etc.), monitor temperature of water inside reservoir and water flow rate of the pipelines, and make the temperature and water flow stable inside the accelerator by controlling the functional components.

4) Accelerator sub-controller: The accelerator of the radiotherapy equipment is installed on the rotating gantry with the function of converting electricity to X-ray. It is composed of pulse modulator, magnetron (including electromagnet), high-power microwave transmission system, accelerator, autofrequency-control system, filament power, gun power and shield. Accelerator sub-controller is responsible for controlling all the functional components mentioned above.

5) Treatment head sub-controller: Four parts of control signals should be generated by the treatment head subcontroller, which are control signals of treatment head rotation motor, flattening filter, primary collimators and MLC linear motors. Due to the large amount (128) and special type (linear motors) of the motors in MLC, control commands and data are not sent to the motors directly, but transit through an MLC control device.

6) Sub-controller of other attachments: Some additional attachments of radiotherapy equipment are needed with the update of treatment methods, such as EPID system and IGRT system. These attachments are usually existing products supplied by other companies, which requires the subcontrollers of these attachments to be consistent with their interface protocols. 


\section{Location Distribution and Communication Mechanism}

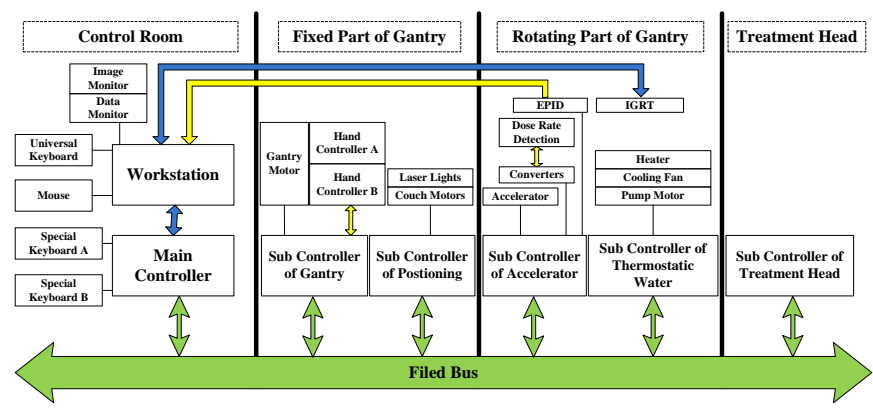

FIGURE II. LOCATION DISTRIBUTION AND COMMUNICATION MECHANISM.

The controllers can be installed in the control room (a separate room from the equipment), fixed part of gantry, rotating part of gantry or treatment head (rotating with the gantry and the head). The principle of the design is to minimize the number of cables and the length of the cable carrier.

Figure 2 shows not only the installation location of each controller, but also the signal transmission communication mechanism among all controllers. As mentioned above, the workstation computer connects with the main controller via network cable (blue in Figure 2). The main controller connects with each sub-controller via field bus (green). The real-time image guidance system connects with the workstation computer via its network interface. As for the electronic portal imaging device system, it not only needs to link with the workstation computer via serial port (yellow), but also a moved axis to control the stretch out and draw back of the panel. In order to simplify the system, the axis is installed on the accelerator controller.

\section{CONTROL FLOW}

The control flow of the radiotherapy equipment differs according to the treatment methods. An example of control flow of static conformal method using the designed DCS is shown in Figure 3.

When the system receives the command of starting treatment, the treatment planning transfer module transfers the treatment planning data into machine language including positions of all axis and dose rate of all position points. When the transfer finished, the system sends the output data to the main controller. Then the main controller sends treatment planning data of the first radiation field to the buffer of all related sub-controllers. After the data is downloaded, motion commands are given to the sub-controllers by the main controller. When all axis arrive at the designated location and the signals are confirmed, beam-on command is given to the sub-controller of accelerator. During the period of beam on, the sub-controller of accelerator determines the dose rate all the time. Once the detected dose error is greater than the given threshold, the beam will be directly cut off by the accelerator sub-controller. The information will be reported to the main controller afterwards. When the beam-on stage ends normally, the main controller sends treatment planning data of next radiation field to the buffer of all related sub-controllers.

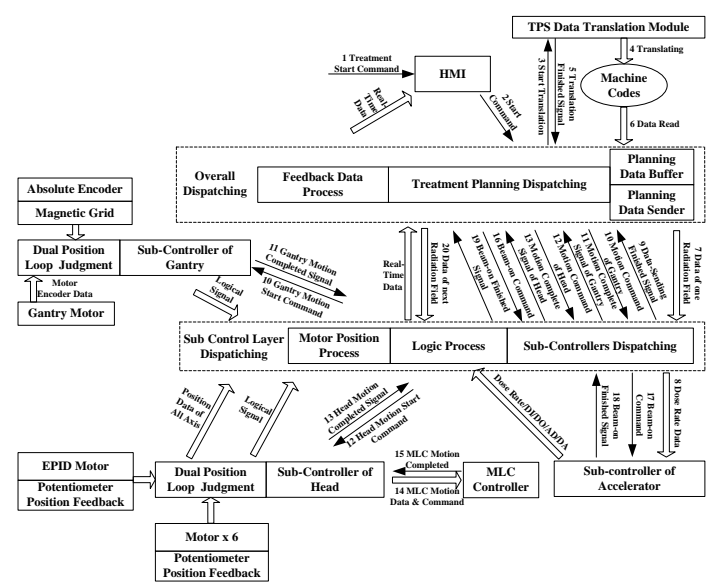

FIGURE III. CONTROL FLOW OF DCS FOR THE RADIOTHERAPY EQUIPMENT.

\section{V.REALIZATION OF DESIGNED DCS FOR RADIOTHERAPY EQUIPMENT}

This article developed a sample machine with DCS for the mentioned radiotherapy equipment as it is shown in Figure 4.

The single cycle time of the main controller is $14.3 \mathrm{~ms}$ when the designed DCS operates under automatic mode. The rotation error of gantry is less than 0.010 and the maximum rotation speed is $36 \mathrm{o} / \mathrm{s}$. The control error of all the motion components in the treatment head is less than $0.01 \mathrm{~mm}$ and the error of radiation fields on central plane is less than $0.025 \mathrm{~mm}$. All the indicators properly meet the requirements of radiation therapy.

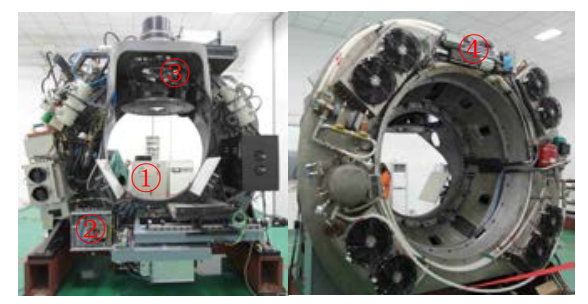

FIGURE IV. SAMPLE MACHINE: 1) ELECTRICAL CABINET WITH GANTRY SUB-CONTROLLER AND PATIENT POSITIONING

SUB-CONTROLLER INSIDE; 2) ACCELERATOR SUBCONTROLLER; 3) HEAD SUB-CONTROLLER; 4) THERMOSTATIC WATER SUB-CONTROLLER.

\section{CONCLUSION}

DCS is widely used among the industry field owing to its advantages of high reliability and easy maintenance. This article designs a DCS for radiotherapy equipments. The specific design thought, module partition, communication mechanism and control flow of this system are given elaborately. With the continuous development of radiotherapy technology, DCS will be more competitive with its capacity to respond to growing number of functional components. It will become an inevitable trend in the development of radiotherapy equipment.

\section{ACKNOWLEDGEMENTS}

This work was supported by Beijing Municipal Science and Technology Project (Z141100000514015), Key Projections of the State Key Lab of Tribology in Tsinghua University 
(SKLT12A03), Independent Interdisciplinary Research Program of Tsinghua University (20111081026) and National Key Technology Support Program (2015BAI03B00).

\section{REFERENCES}

[1] Bragg C. Adaptive motion compensation in radiotherapy. Cambridge University Press: Cambridge, 2013.

[2] McNair H.A., Elsworthy M., Dean J. \& Beardmore C., Image guided radiotherapy: Current status of soft tissue imaging. Radiography. 20(2), pp.158-161, 2014.

[3] Sawant A., Venkat R. \& Srivastava V., Management of threedimensional intrafraction motion through real-time DMLC tracking. Medical Physics. 35(5), pp.2050-2056, 2008.

[4] Mattick W.L., Radiation Therapy of Cancer1: Basic Principles, Their Application and Results. Radiology. 18(6), pp.1070-1089, 1932.

[5] Shi S.H., Fan W.G. \& Ye P.Q., Five Axis CNC System Based on RTLinux for Electrical Discharge Grinding Polycrystalline Diamond Tools. Journal of Basic Science and Engineering. 01, pp.179-188, 2014. (in Chinese)

[6] Zhang ZF, Shuai M, Wei H. Design and Realization of Humanoid Robot Distributed Control System. Computer Engineering. 2011(16):247-250. (in Chinese) 